ALEA, Lat. Am. J. Probab. Math. Stat. 18, 1085-1102 (2021)

DOI: $10.30757 /$ ALEA.v18-41

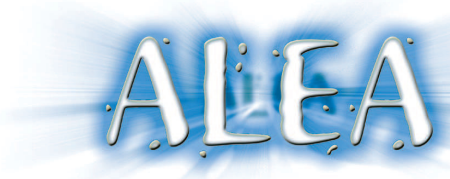

\title{
A CLT for a class of stochastic integrals with application in statistics
}

\section{Johanna Garzón, Jaime San Martín and Soledad Torres}

Departamento de Matemáticas, Universidad Nacional de Colombia, Bogotá, Colombia.

E-mail address: mjgarzonm@unal.edu.co

CMM-DIM, UMI-CNRS 2807

Universidad de Chile,

Casilla 170-3 Correo 3

Santiago, Chile.

E-mail address: jsanmart@dim.uchile.cl

CIMFAV - Facultad de Ingeniería,

Universidad de Valparaíso,

Valparaíso, Chile.

E-mail address: soledad.torres@uv.cl

Abstract. In this article we give a direct proof of a central limit theorem and law of large numbers for a functional with compact support of a diffusion. Some applications are given in order to obtain a parameter estimation for different models.

\section{Introduction}

Given a diffusion $X$ solution of the Stochastic Differential Equations (SDE)

$$
X_{t}=X_{0}+B_{t}+\int_{0}^{t} h\left(X_{s}\right) d s
$$

where $h$ is a smooth function with compact support and $B$ is a Brownian motion $(\mathbf{B m})$. We want to give the asymptotic distribution of the following functional of $X$

$$
F_{t}=\int_{0}^{t} g\left(X_{s}\right) d B_{s}
$$

Received by the editors September 22th, 2020; accepted March 24th, 2021.

2010 Mathematics Subject Classification. 60F05, 60K05.

Key words and phrases. Central Limit Theorem, Skew Brownian motion, Renewal Process.

The authors acknowledge the support of the Basal project AFB170001. The third author was partially supported by the MATHAMSUD 19-MATH-06 Project, Fondecyt Grant 1171335 and CONICYT - MATHAMSUD FANTASTIC Código 20-MATH-05. 
where we also assume that $g \neq 0$ is compactly supported. Without a loss of generality we shall assume that both $h, g$ have a support contained in $[-1,1]$. The main tool we shall use is to study the trajectory of $\mathrm{X}$ upon the renovation times where it hits \pm 1 .

Existence and uniqueness of this type of equations can be found for example in Takanobu (1986) and limit theorems for stochastic integrals related to solutions of SDEs have been studied for a long time, and there is an extensive literature concerning this subject (see e.g. Basawa and Prakasa Rao, 1980; Delattre and Jacod, 1997; Jacod and Shiryaev, 2003; Khasminskii, 2001; Kurtz and Protter, 1991; Kutoyants, 2004; Prakasa Rao, 1999; Prakasa Rao and Rubin, 1979, and the references therein). In most cases, these works are related to the study of stability for solutions of stochastic differential equations or the study of discrete-time schemes for diffusion processes.

If $X=B$, that is $h \equiv 0$, then in Revuz and Yor (1991); Delattre and Hoffmann (2002) by the Papanicolaou-Strook-Varadhan we can obtain, the following limit (in distribution)

$$
\left(T^{-1 / 4} \int_{0}^{T} g\left(B_{s}\right) d B_{s}, T^{-1 / 2} \int_{0}^{T} g^{2}\left(B_{s}\right) d s\right) \rightarrow\left(B_{\int g^{2}(x) d x L_{1}^{0}(\beta)}, \int g^{2}(x) d x L_{1}^{0}(\beta)\right),
$$

where $(B, \beta)$ are two independent Brownian motions and $L_{t}^{x}(\beta)$ denotes the Local time of $\beta$ at time $t$ at level $x$. Our result bears some similarity with this one.

Khasminskii (2001) (see also Kutoyants, 2004) is the closest result with our work, nevertheless the main difference with ours is that the author considers only martingale diffusions $Y=\left(Y_{s}\right)$ and the results obtained are the law of large numbers (LLN) and the central limit theorem (CLT) with standard normalization $\left(T\right.$ and $\sqrt{T}$ ) for the Riemann integral $\int f\left(Y_{s}\right) d s$. Although, our main Theorem 2.4 can be obtained using a combination of results from Höpfner and Löcherbach (2003), which are based upon some classical results from Khasminskii (2001) and Khasminskii (2012), putting these arguments together is tricky and does not allow us to fully understand which are the main points of this proof. We propose in this article a new and direct construction based on renovation theory that provides insights on the main technical points, a clear explanation on the limiting distribution for path functionals like (1.2), and could be used to effectively simulate and do numerical analysis on them, with possible applications that we discuss at the end of the article, some of them are up to our knowledge novel. The new technical tools we develop in this article are Lemma 2.1, Lemma 2.3 and Theorem 2.2.

This paper is organized as follows. Section 2 introduces some properties about the trajectory of $X$ of (1.1) and the main results of the paper. The proofs are given in the section 3 . Section 4 is devoted to some applications of those results to simulate paths of functionals of $X$ and to estimate the parameter of the Skew Brownian motion and nonparametric estimation of the drift in SDE. Finally, Section 5 presents a list of notations in order to facilitate the reading of this article.

\section{Main Results}

In this section we will study the trajectory of $X$ of (1.1) upon the renovation times where it hits \pm 1 . For that purpose we define the following stopping times:

$$
\begin{aligned}
& T_{0}=\inf \left\{t \geq 0: X_{t} \in\{-1,1\}\right\}, \mathscr{T}_{0}=T_{0}, \quad R_{0}=\mathscr{R}_{0}=0 \\
& R_{i+1}=\inf \left\{t>0: X_{t+\mathscr{T}_{i}} \in\{-2,2\}\right\}, \quad \mathscr{R}_{i+1}=\mathscr{T}_{i}+R_{i+1} \\
& T_{i+1}=\inf \left\{t>0: X_{t+\mathscr{R}_{i+1}} \in\{-1,1\}\right\}, \quad \mathscr{T}_{i+1}=\mathscr{R}_{i+1}+T_{i+1}
\end{aligned}
$$

First, we shall assume that $X_{0}$ has initial distribution supported on $\{-1,1\}$, where $\mathbb{P}\left(X_{0}=\right.$ 1) $=p=1-\mathbb{P}\left(X_{0}=-1\right)=1-q$, in such a way that the successive renovations have the same distribution. Then we shall consider general initial conditions (see subsection 3.5). The renovation times are $\mathscr{T}_{i}$ at which the process $X$ is at \pm 1 . This defines a Markov chain $Z_{n}=X_{\mathscr{T}_{n}}$. Thus, 


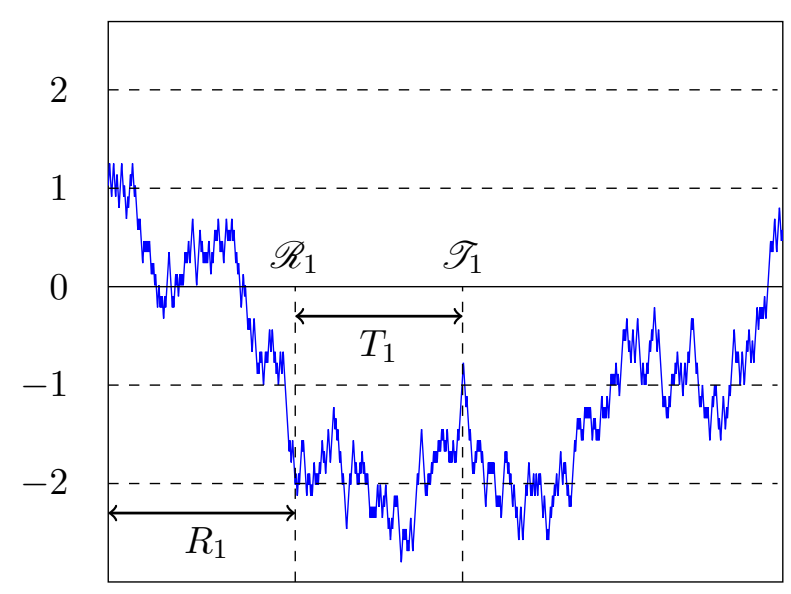

Figure 2.1. Diffusion $X$ starting at 1.

$p=\mathbb{P}\left(Z_{0}=1\right), q=\mathbb{P}\left(Z_{0}=-1\right)$ is the stationary distribution of $\left(Z_{n}\right)$. The transition kernel for this Markov chain is

$$
P=\left(\begin{array}{cc}
\alpha & 1-\alpha \\
1-\beta & \beta
\end{array}\right)
$$

where $0<\alpha=\mathbb{P}_{1}\left(X_{R_{1}}=2\right)<1 ; 0<\beta=\mathbb{P}_{-1}\left(X_{R_{1}}=-2\right)<1$. We obtain that

$$
p=\frac{1-\beta}{2-(\alpha+\beta)}, q=\frac{1-\alpha}{2-(\alpha+\beta)} .
$$

In the sequel, unless we specify the contrary, $\mathbb{P}$ denotes the probability law assuming that $X_{0}$ has this special distribution.

We point out that $\left\{\left(T_{i}\right): i \geq 1\right\}$ are identically distributed as the hitting time of 1 for a standard $\mathrm{Bm}$. They are also independent, but this is not so obvious (see Lemma 2.1). However, the random times $\left(R_{i}: i \geq 1\right)$ are neither independent, nor identically distributed. We shall decompose them into independent components as follows:

$$
R_{i}=R_{i,-1}\left(1-Z_{i-1}\right) / 2+R_{i, 1}\left(Z_{i-1}+1\right) / 2
$$

which simply says that when $Z_{i-1}=1$ we have $R_{i}=R_{i, 1}$ and when $Z_{i-1}=-1$, we have $R_{i}=R_{i,-1}$. The collections of random times $\left(R_{i, 1}\right)$ and $\left(R_{i,-1}\right)$ are independent and each collection is i.i.d., distributed as

$$
\begin{gathered}
R_{i, 1} \stackrel{\mathrm{d}}{=} \inf \left\{t>0: X_{t} \in\{-2,2\}\right\}, \text { when } X_{0}=1 ; \\
R_{i,-1} \stackrel{\mathrm{d}}{=} \inf \left\{t>0: X_{t} \in\{-2,2\}\right\}, \text { when } X_{0}=-1 .
\end{gathered}
$$

In order to have pointwise equality in (2.3), for $i \geq 0$ we define $X^{i, \pm 1}$ as the unique solutions of

$$
X_{t}^{i, \pm 1}= \pm 1+B_{\mathscr{T}_{i}+t}-B_{\mathscr{T}_{i}}+\int_{0}^{t} h\left(X_{s}^{i, \pm 1}\right) d s
$$

Then, we define

$$
R_{i+1, \pm 1}=\inf \left\{t>0: X_{t}^{i, \pm 1} \in\{-2,2\}\right\}
$$

Notice we have $\left(R_{j+1, \pm 1}\right)_{j \geq i}$ are independent of $\left(Z_{n}\right)_{n \leq i}$. We have $X_{t}=X_{t-\mathscr{T}_{i}}^{i, Z_{i}}$ that holds when $t \in\left[\mathscr{T}_{i}, \mathscr{T}_{i+1}\right)$.

We consider the renewal process associated to $\left(\mathscr{T}_{i}\right)$, given by

$$
N_{t}=\max \left\{n: \mathscr{T}_{n} \leq t\right\}
$$


As an auxiliary renewal process we consider

$$
\mathscr{S}_{n}=\sum_{i=0}^{n} T_{i}, \quad M_{t}=\max \left\{n: \mathscr{S}_{n} \leq t\right\} .
$$

Now, we establish some important results about the hitting times and renewal processes defined before.

\section{Lemma 2.1.}

(i) The random times $\left(T_{i}\right)$ are independent and they are also independent of $\left(Z_{n}\right)$.

(ii) For every $n$

$$
\mathscr{S}_{n} \stackrel{d}{=} \inf \left\{t>0: B_{t}=n\right\} .
$$

(iii) There exists a Brownian motion $W$ such that for all $t$

$$
M_{t}=\left\lfloor\max _{s \leq t} W_{s}\right\rfloor .
$$

Theorem 2.2. For all $t$ we have $N_{t} \leq M_{t}$ and

$$
\lim _{t \rightarrow \infty} \mathbb{P}\left(N_{t}<M_{t}\right)=0 .
$$

Lemma 2.3. The process $\left(M_{t}\right)_{t}$ is independent of the excursions $\left(X_{\mathscr{T}_{i}+s}: 0 \leq s \leq R_{i+1}\right)_{i}$.

The following theorem give important results to obtain the asymptotic distribution of (1.2) and the parameter estimation in the skew Brownian motion $(\mathrm{sBm})$ case.

Theorem 2.4. The following properties hold

(i) $M_{t}$ (and therefore $N_{t}$ ) has the following limit in distribution

$$
\lim _{t \rightarrow \infty} \frac{M_{t}}{\sqrt{t}} \stackrel{d}{=} \Gamma
$$

where $\Gamma$ has the distribution of the absolute value of a $N(0,1)$.

(ii) The asymptotic distribution for $F_{t}$ given by (1.2) is

$$
\lim _{t \rightarrow \infty} \frac{\int_{0}^{t} g\left(X_{s}\right) d B_{s}}{t^{1 / 4}} \stackrel{d}{=} \sigma \sqrt{\Gamma} Y
$$

where $\Gamma$ is distributed as before, $Y$ is a $N(0,1)$, they are independent and

$$
\sigma^{2}=\mathbb{E}_{X_{0}}\left(\int_{0}^{R_{1}} g^{2}\left(X_{s}\right) d s\right) .
$$

Here $X_{0}$ has initial distribution $\mathbb{P}\left(X_{0}=1\right)=p, \mathbb{P}\left(X_{0}=-1\right)=q$.

(iii) The weak law of large numbers

$$
\lim _{t \rightarrow \infty} \frac{\int_{0}^{t} g^{2}\left(X_{s}\right) d s}{M_{t}} \stackrel{\mathbb{P}}{=} \sigma^{2} .
$$

(iv) Finally,

where $Y$ is a $N(0,1)$.

$$
\lim _{t \rightarrow \infty} \frac{\int_{0}^{t} g\left(X_{s}\right) d B_{s}}{\sqrt{\int_{0}^{t} g^{2}\left(X_{s}\right) d s}} \stackrel{d}{=} Y
$$

Remark 2.5. Note that in $(i i), \sqrt{\Gamma} Y$ has the same distribution as $\left(B_{\max _{0 \leq s \leq}\left(W_{s}\right)}\right)$, where $W$ is a Brownian motion independent of $B$; which corresponds to a particular case of the Mittag-Leffler distribution (see Höpfner and Löcherbach, 2003). 


\section{Proofs of the results}

3.1. Proof of Lemma 2.1. In what follows we denote by $\left(\mathcal{F}_{t}\right)$ the natural filtration of $\left(B_{t}\right)$.

(i) Let us introduce the following Markov chain $V_{n}=X_{\mathscr{R}_{n}}: n \geq 1$, which takes values in $\{-2,2\}$. Let us show that $V_{n}=2 Z_{n}$ for all $n \geq 1$. Indeed, we have

$$
Z_{n}=X_{\mathscr{T}_{n}}=X_{\mathscr{R}_{n}}+\left(-\operatorname{sign}\left(X_{\mathscr{R}_{n}}\right)\right)=\frac{1}{2} X_{\mathscr{R}_{n}}=\frac{1}{2} V_{n}
$$

In particular $\mathbb{P}\left(V_{n}=2\right)=p, \mathbb{P}\left(V_{n}=-2\right)=q$. In what follows we denote by $T$ the hitting time of 1 for a standard Bm.

Now, we show the desired property by induction. So let us start with $T_{1}$ and $Z_{1}$. Notice that $X_{\mathscr{R}_{1}+t}-X_{\mathscr{R}_{1}}=B_{\mathscr{R}_{1}+t}-B_{\mathscr{R}_{1}}$, holds for all $0 \leq t \leq T_{1}$. Therefore, we have

$$
T_{1}=\inf \left\{t \geq 0: B_{\mathscr{R}_{1}+t}-B_{\mathscr{R}_{1}}=-V_{1} / 2\right\} \text {. }
$$

The main point here is that $B_{\mathscr{R}_{1}+t}-B_{\mathscr{R}_{1}}$ is independent of $V_{1}$. This implies that $\mathbb{P}\left(T_{1} \in d s, V_{1}=\right.$ $2)=p \mathbb{P}(T \in d s)$ and $\mathbb{P}\left(T_{1} \in d s, V_{1}=-2\right)=q \mathbb{P}(T \in d s)$. This shows the desired independence.

We shall prove now the independence of $T_{1}, T_{2},\left(Z_{1}, Z_{2}\right)$. Recall that

$$
\begin{aligned}
& T_{1}=\inf \left\{t \geq 0: B_{\mathscr{R}_{1}+t}-B_{\mathscr{R}_{1}}=-V_{1} / 2\right\}, Z_{1}=V_{1} / 2, \\
& T_{2}=\inf \left\{t \geq 0: B_{\mathscr{R}_{2}+t}-B_{\mathscr{R}_{2}}=-V_{2} / 2\right\}, Z_{2}=V_{2} / 2 .
\end{aligned}
$$

In particular $\left(Z_{1}, Z_{2}, T_{2}\right)$ is measurable with respect to $\sigma\left(Z_{1}, B_{\mathscr{T}_{1}+\bullet}-B_{\mathscr{T}_{1}}\right)$. Hence, $T_{1}$ is independent of $\left(Z_{1}, Z_{2}, T_{2}\right)$. On the other hand, $\mathbb{P}\left(T_{2} \in d s, Z_{1}=z_{1}, Z_{2}=z_{2}\right)=\mathbb{P}(T \in d s) \mathbb{P}\left(Z_{1}=\right.$ $\left.z_{1}, Z_{2}=z_{2}\right)$. This shows the desired independence. The rest of the proof is done by induction.

(ii), (iii) Consider the process

$$
\begin{aligned}
W_{t} & =\sum_{i=1}^{M_{t}} \frac{-V_{i}}{2}\left(B_{\mathscr{T}_{i}}-B_{\mathscr{R}_{i}}\right)+\frac{-V_{M_{t}+1}}{2}\left(B_{\mathscr{R}_{M_{t}+1}+\left(t-\mathscr{S}_{M_{t}}\right)}-B_{\mathscr{R}_{M_{t}+1}}\right) \\
& =M_{t}+\frac{-V_{M_{t}+1}}{2}\left(B_{\mathscr{R}_{M_{t}+1}+\left(t-\mathscr{S}_{M_{t}}\right)}-B_{\mathscr{R}_{M_{t}+1}}\right) \\
& =\sum_{n}\left(n+\frac{-V_{n+1}}{2}\left(B_{\mathscr{R}_{n+1}+\left(t-\mathscr{S}_{n}\right)}-B_{\mathscr{R}_{n+1}}\right)\right) 1_{\mathscr{S}_{n} \leq t<\mathscr{S}_{n+1}},
\end{aligned}
$$

which is simply the sum of the Bm excursions $\Xi^{i}=\left(B_{\mathscr{R}_{i}+s}-B_{\mathscr{R}_{i}}: 0 \leq s \leq T_{i}\right)$ modulated by the sign of $-V_{i}$. Let us prove that all these excursions are independent, and independent of $\left(V_{n}\right)_{n}$. We do this for $\Xi^{1}, \Xi^{2}, V_{1}, V_{2}$, and the general case follows from induction.

Notice that $\Xi^{2}$ is independent of $\mathcal{F}_{\mathscr{R}_{2}}$, in particular we have

$$
\mathbb{P}\left(\Xi^{1} \in \mathcal{A}_{1}, V_{1}=v_{1}, V_{2}=v_{2}, \Xi^{2} \in \mathcal{A}_{2}\right)=\mathbb{P}\left(\Xi^{1} \in \mathcal{A}_{1}, V_{1}=v_{1}, V_{2}=v_{2}\right) \mathbb{P}\left(\Xi^{2} \in \mathcal{A}_{2}\right) .
$$

The key observation is that $V_{1}, V_{2}$ is measurable in $\sigma\left(V_{1}, B_{\mathscr{T}_{1}+\bullet}-B_{\mathscr{T}_{1}}\right)$. So, it is enough to show that $\Xi^{1}$ is independent of $V_{1}, B_{\mathscr{T}_{1}+\bullet}-B_{\mathscr{T}_{1}}$

$$
\begin{aligned}
& \mathbb{P}\left(\Xi^{1} \in \mathcal{A}_{1}, V_{1}=v_{1}, B_{\mathscr{T}_{1}+\bullet}-B_{\mathscr{T}_{1}} \in \mathcal{C}\right)=\mathbb{P}\left(\Xi^{1} \in \mathcal{A}_{1}, V_{1}=v_{1}\right) \mathbb{P}\left(B_{\mathscr{T}_{1}+\bullet}-B_{\mathscr{T}_{1}} \in \mathcal{C}\right) \\
& =\mathbb{P}\left(\Xi^{1} \in \mathcal{A}_{1}\right) \mathbb{P}\left(V_{1}=v_{1}\right) \mathbb{P}\left(B_{\mathscr{T}_{1}+\bullet}-B_{\mathscr{T}_{1}} \in \mathcal{C}\right) .
\end{aligned}
$$

This shows the claim. Now, it is straightforward to show that $W$ is a standard Bm. Also, it is direct to prove that

$$
\mathscr{S}_{n}=\inf \left\{t>0: W_{t}=n\right\} .
$$

From here we deduce that $M_{t}=\left\lfloor\max _{s \leq t} W_{s}\right\rfloor$. 
3.2. Proof of Theorem 2.2. In what follows we denote by $\mathscr{M}_{t}$ the running maximum of $W$, that is

$$
\mathscr{M}_{t}=\max _{0 \leq s \leq t} W_{s} .
$$

It is clear that $N_{t} \leq M_{t}$. Now consider the random variables $\widehat{R}_{i}=R_{i, 1}+R_{i,-1}$. These random variables are positive and i.i.d.. They have exponential moments, that is, there exists $\lambda>0$ such that $\mathbb{E}\left(e^{\lambda \widehat{R}_{1}}\right)<\infty$, so they have finite moments. In particular, they satisfy the strong law of large numbers

$$
\lim _{n \rightarrow \infty} \frac{1}{n} \sum_{i=1}^{n} \widehat{R}_{i}=\mathbb{E}\left(\widehat{R}_{1}\right)
$$

where the limit holds a.s..

Therefore, the random variable $\Psi=\sup \left\{\frac{1}{n} \sum_{i=1}^{n} \widehat{R}_{i}: n \geq 1\right\}$ is finite a.s.

On the other hand, if $1 / 2<\gamma<1$ then

$$
\lim _{t \rightarrow \infty} \frac{\Psi M_{t}}{t^{\gamma}} \stackrel{\mathrm{d}}{=} 0
$$

which implies that the limit also holds in probability.

Assume now that $\Psi\left(M_{t}+1\right)<t^{\gamma}$, then we have

$$
t<\sum_{i=1}^{N_{t}+1} T_{i}+R_{i}<\sum_{i=1}^{N_{t}+1} T_{i}+\widehat{R}_{i} \leq \sum_{i=1}^{N_{t}+1} T_{i}+\Psi\left(M_{t}+1\right) \leq \sum_{i=1}^{N_{t}+1} T_{i}+t^{\gamma} .
$$

From these inequalities, we conclude that

$$
t-t^{\gamma}<\sum_{i=1}^{N_{t}+1} T_{i}
$$

which implies that $M_{t-t^{\gamma}}<N_{t}+1$. Given that $N_{t}$ is an integer valued we conclude that

$$
\mathscr{M}_{t-t^{\gamma}}-1 \leq N_{t} \leq M_{t}<\mathscr{M}_{t} \text {. }
$$

The last strict inequality holds a.s., for fixed $t$. The important conclusion is that

$$
M_{t}-N_{t}<\mathscr{M}_{t}-\mathscr{M}_{t-t \gamma}+1 \text {. }
$$

Let us now show that $\mathbb{P}\left(\mathscr{M}_{t}-\mathscr{M}_{t-t^{\gamma}}>0\right)$ converges to 0 . Notice that this is stronger than proving that $\mathscr{M}_{t}-\mathscr{M}_{t-t^{\gamma}}$ converges in probability to 0 . The claimed limit follows from scaling properties of Bm. In fact, since

$$
\mathscr{M}_{t}-\mathscr{M}_{t-t^{\gamma}} \stackrel{\mathrm{d}}{=} \sqrt{t}\left(\mathscr{M}_{1}-\mathscr{M}_{1-t^{\gamma-1}}\right)
$$

we deduce

$$
\begin{aligned}
\mathbb{P}\left(\mathscr{M}_{t}-\mathscr{M}_{t-t^{\gamma}}>0\right) & =\mathbb{P}\left(\mathscr{M}_{1}-\mathscr{M}_{1-t^{\gamma-1}}>0\right)=\mathbb{P}\left(\tau>1-t^{\gamma-1}\right) \\
& =\int_{1-t^{\gamma-1}}^{1} \frac{d s}{\pi \sqrt{s(1-s)}},
\end{aligned}
$$

where $\tau \in[0,1]$ is the time where $\mathscr{M}_{1}$ is attained. Then, this probability clearly converges to 0 .

Finally,

$$
\begin{aligned}
\mathbb{P}\left(M_{t}>N_{t}\right) & =\mathbb{P}\left(M_{t}>N_{t}, \Psi M_{t}<t^{\gamma}\right)+\mathbb{P}\left(M_{t}>N_{t}, \Psi M_{t} \geq t^{\gamma}\right) \\
& \leq \mathbb{P}\left(M_{t}>N_{t}, \Psi M_{t}<t^{\gamma}\right)+\mathbb{P}\left(\Psi M_{t} \geq t^{\gamma}\right) \\
& \leq \mathbb{P}\left(1 \leq M_{t}-N_{t}<\mathscr{M}_{t}-\mathscr{M}_{t-t^{\gamma}}+1, \Psi M_{t}<t^{\gamma}\right)+\mathbb{P}\left(\Psi M_{t} \geq t^{\gamma}\right) \\
& \leq \mathbb{P}\left(\mathscr{M}_{t}-\mathscr{M}_{t-t \gamma}>0\right)+\mathbb{P}\left(\Psi M_{t} \geq t^{\gamma}\right),
\end{aligned}
$$

which converges to 0 and the result is shown. 
3.3. Proof of Lemma 2.3. The proof that follows is in the spirit of the proof of Lemma 2.1. For $z \in\{-1,1\}$ consider $X^{i, z}$ the unique solution of the SDE given in (2.4). We also recall the definition of $R_{i+1, z}$. Similarly, we define $T_{i, z}$ as

$$
T_{i, z}=\inf \left\{t>0: B_{\mathscr{R}_{i}+t}-B_{\mathscr{R}_{i}}=z\right\} .
$$

We also define $T_{0, z}=0$. In order to show that $\left(T_{k}\right)_{k}$ and $\left(X_{\mathscr{T}_{i}+s}: 0 \leq s \leq R_{i+1}\right)_{i}$ are independent it is enough to show that $\left(T_{k}\right)_{k}$ and $\left\{\left(Z_{n}\right)_{n},\left(B_{\mathscr{T}_{i}+s}-B_{\mathscr{T}_{i}}: 0 \leq s \leq R_{i+1}\right)_{i}\right\}$ are independent. For that purpose we define $B_{s}^{k}=B_{\mathscr{T}_{k}+s}-B_{\mathscr{T}_{k}}$ and consider (we take $A_{0}=\{0\}$ )

$$
\begin{aligned}
& \mathbb{P}\left(T_{k} \in A_{k},\left(B_{s}^{k}: 0 \leq s \leq R_{k+1}\right) \in \mathcal{A}_{k}, Z_{k}=z_{k}, k=0, \cdots, m\right) \\
= & \mathbb{P}\left(T_{k, z_{k}} \in A_{k},\left(B_{s}^{k}: 0 \leq s \leq R_{k+1, z_{k}}\right) \in \mathcal{A}_{k}, Z_{k}=z_{k}, k=0, \cdots, m\right) \\
= & \mathbb{P}\left(T_{k, z_{k}} \in A_{k},\left(B_{s}^{k}: 0 \leq s \leq R_{k+1, z_{k}}\right) \in \mathcal{A}_{k}, Z_{k}=z_{k}, k=0, \cdots, m-1, Z_{m}=z_{m}\right) \\
& \times \mathbb{P}\left(\left(B_{s}^{m}: 0 \leq s \leq R_{m+1, z_{m}}\right) \in \mathcal{A}_{m}\right) \mathbb{P}\left(T_{m, z_{m}} \in A_{m}\right) \\
= & \mathbb{P}\left(T_{k, z_{k}} \in A_{k},\left(B_{s}^{k}: 0 \leq s \leq R_{k+1, z_{k}}\right) \in \mathcal{A}_{k}, Z_{k}=z_{k}, k=0, \cdots, m-1, Z_{m}=z_{m}\right) \\
& \times \mathbb{P}\left(\left(B_{s}^{m}: 0 \leq s \leq R_{m+1, z_{m}}\right) \in \mathcal{A}_{m}\right) \mathbb{P}\left(T_{m} \in A_{m}\right)
\end{aligned}
$$

Note that if $Z_{m-1}=z_{m-1}$ then $Z_{m}=z_{m}$ iff $X_{\mathscr{T}_{m-1}+R_{m, z_{m-1}}}-X_{\mathscr{T}_{m-1}}=2 z_{m}-z_{m-1}$. The increment $X_{\mathscr{T}_{m-1}+R_{m, z_{m-1}}}-X_{\mathscr{T}_{m-1}}$ is a functional of $B^{m-1}$. So, we can include this restriction to have $Z_{m-1}=z_{m-1},\left(B_{s}^{m-1}: 0 \leq s \leq R_{m, z_{m-1}}\right) \in \mathcal{A}_{m-1}, Z_{m}=z_{m}$ is equivalent to $Z_{m-1}=$ $z_{m-1}, B_{\bullet}^{m-1} \in \widetilde{\mathcal{A}}_{m-1}$.

Then, we can continue to conclude

$$
\begin{aligned}
& \mathbb{P}\left(T_{k} \in A_{k},\left(B_{s}^{k}: 0 \leq s \leq R_{k+1}\right) \in \mathcal{A}_{k}, Z_{k}=z_{k}, k=0, \cdots, m\right) \\
= & \mathbb{P}\left(T_{k, z_{k}} \in A_{k},\left(B_{s}^{k}: 0 \leq s \leq R_{k+1, z_{k}}\right) \in \mathcal{A}_{k}, Z_{k}=z_{k}, k=0, \cdots, m-2,\right. \\
& \left.\quad Z_{m-1}=z_{m-1}\right) \mathbb{P}\left(B_{\bullet}^{m-1} \in \widetilde{\mathcal{A}}_{m-1}\right) \mathbb{P}\left(T_{m-1, z_{m-1}} \in A_{m-1}\right) \\
& \times \mathbb{P}\left(\left(B_{s}^{m}: 0 \leq s \leq R_{m+1, z_{m}}\right) \in \mathcal{A}_{m}\right) \times \mathbb{P}\left(T_{m} \in A_{m}\right) \\
= & \mathbb{P}\left(T_{k, z_{k}} \in A_{k},\left(B_{s}^{k}: 0 \leq s \leq R_{k+1, z_{k}}\right) \in \mathcal{A}_{k}, Z_{k}=z_{k}, k=0, \cdots, m-2,\right. \\
& \left.Z_{m-1}=z_{m-1}\right) \mathbb{P}\left(B_{\bullet}^{m-1} \in \widetilde{\mathcal{A}}_{m-1}\right) \mathbb{P}\left(\left(B_{s}^{m}: 0 \leq s \leq R_{m+1, z_{m}}\right) \in \mathcal{A}_{m}\right) \\
& \times \mathbb{P}\left(T_{m} \in A_{m}\right) \mathbb{P}\left(T_{m-1} \in A_{m-1}\right) .
\end{aligned}
$$

Then the claim follows by induction.

3.4. Proof of Theorem 2.4. First we introduce some new variables and establish some auxiliary lemmas.

We consider the variables

$$
\psi_{i, z}=\int_{0}^{R_{i+1, z}} g\left(X_{s}^{i, z}\right) d B_{s}^{i},
$$

where $B_{s}^{i}=B_{\mathscr{T}_{i}+s}-B_{\mathscr{T}_{i}}$. We point out that

$$
\psi_{i}=: \int_{\mathscr{T}_{i}}^{\mathscr{R}_{i+1}} g\left(X_{s}\right) d B_{s}=\psi_{i, Z_{i}} .
$$

Our first tool is the following lemma. 
Lemma 3.1. The following CLT holds for $\left(\psi_{i}\right)$

$$
\lim _{n \rightarrow \infty} \frac{1}{\sqrt{n}} \sum_{i=0}^{n} \psi_{i} \stackrel{d}{=} \sigma Y
$$

where $Y$ is distributed as a $N(0,1)$.

Proof: $\psi_{i}$ is a mixture of two distribution $\psi_{i, 1}$, and $\psi_{i,-1}$ driven by the stationary Markov chain $Z$ which takes two values $\{1,-1\}$, where $\left\{\psi_{i, 1}\right\}$ (respectively $\left\{\psi_{i,-1}\right\}$ ) is a set of i.i.d random variables with finite variance and independent of $Z$. A direct computation of the characteristic function of $\frac{1}{\sqrt{n}} \sum_{i=0}^{n} \psi_{i}$ conditioning on $Z$ gives the result.

\section{Lemma 3.2.}

$$
\lim _{t \rightarrow \infty} \frac{1}{t^{1 / 4}} \sum_{i=0}^{N_{t}} \psi_{i} \stackrel{d}{=} \sigma \sqrt{\Gamma} Y
$$

where $Y$ is distributed as a $N(0,1), \Gamma$ has the distribution of the absolute value of a $N(0,1)$ and both are independent.

Proof: For every $x \in \mathbb{R}$ we have

$$
\left|\mathbb{P}\left(\frac{1}{t^{1 / 4}} \sum_{i=0}^{N_{t}} \psi_{i} \leq x\right)-\mathbb{P}\left(\frac{1}{t^{1 / 4}} \sum_{i=0}^{M_{t}} \psi_{i} \leq x\right)\right| \leq 2 \mathbb{P}\left(N_{t}<M_{t}\right) .
$$

So, it is enough to show the result for $M_{t}$ instead of $N_{t}$. On the other hand, according to Lemma 2.3 $M_{t}$ is independent of $\left(\psi_{i}\right)_{i}$ and according to Lemma 2.1 $M_{t} \stackrel{\mathrm{d}}{=}\lfloor\sqrt{t} \Gamma\rfloor$. Therefore, if we further assume that $\Gamma$ is independent of $(\psi)_{i}$, we get

$$
\begin{aligned}
\mathbb{P}\left(\frac{1}{t^{1 / 4}} \sum_{i=0}^{M_{t}} \psi_{i} \leq x\right) & =\mathbb{P}\left(\frac{1}{t^{1 / 4}} \sum_{i=0}^{\lfloor\sqrt{t} \Gamma\rfloor} \psi_{i} \leq x\right)=\mathbb{P}\left(\frac{\sqrt{\Gamma}}{(\sqrt{t} \Gamma)^{1 / 2}} \sum_{i=0}^{\lfloor\sqrt{t} \Gamma\rfloor} \psi_{i} \leq x\right) \\
& =\int_{0}^{\infty} \mathbb{P}\left(\frac{1}{(\sqrt{t} w)^{1 / 2}} \sum_{i=0}^{\lfloor\sqrt{t} w\rfloor} \psi_{i} \leq \frac{x}{\sqrt{w}}\right) \mathbb{P}(\Gamma \in d w) .
\end{aligned}
$$

The Dominated Convergence Theorem, Lemma 3.2 and the fact that $\mathbb{P}(\Gamma>0)=1$, shows that

$$
\lim _{t \rightarrow \infty} \mathbb{P}\left(\frac{1}{t^{1 / 4}} \sum_{i=0}^{M_{t}} \psi_{i} \leq x\right)=\int_{0}^{\infty} \mathbb{P}(\sigma Y \leq x / \sqrt{w}) \mathbb{P}(\Gamma \in d w),
$$

and the result is shown.

The same technique allows us to show the following result

Lemma 3.3. Let $\phi_{i}=\int_{\mathscr{T}_{i}}^{\mathscr{R}_{i+1}} g^{2}\left(X_{s}\right) d s$, then

$$
\lim _{t \rightarrow \infty} \frac{1}{M_{t}} \sum_{i=0}^{N_{t}} \phi_{i} \stackrel{\mathbb{P}}{=} \sigma^{2}
$$

Now, we are in a position to show Theorem 2.4. 
Proof: $(i)$ This property follows immediately from Lemma $2.2(i i i)$ and the fact that $\max _{s \leq t} W_{s} \stackrel{\mathrm{d}}{=} \sqrt{t} \Gamma$.

(ii) Notice that

$$
\int_{0}^{t} g\left(X_{s}\right) d B_{s}=\sum_{i=0}^{N_{t}} \psi_{i}+\int_{\mathscr{T}_{N_{t}}}^{t} g\left(X_{s}\right) d B_{s}=\sum_{i=0}^{N_{t}} \psi_{i}+\mathcal{E}_{t} .
$$

Of course $t \in\left[\mathscr{T}_{N_{t}}, \mathscr{T}_{N_{t}+1}\right)$. The term $\mathcal{E}_{t}$ is also expressed as

$$
\mathcal{E}_{t}=\int_{\mathscr{T}_{N_{t}}}^{t} g\left(X_{s}\right) d B_{s}=\int_{\mathscr{T}_{N_{t}}}^{t \wedge \mathscr{R}_{N_{t}}} g\left(X_{s}\right) d B_{s}
$$

Using Doob's inequality we get that

$$
\mathbb{E}\left(\mathcal{E}_{t}^{2}\right) \leq C \sigma^{2}
$$

In particular, $\frac{\mathcal{E}_{t}}{t^{1 / 4}}$ converges to 0 in Probability. Then, the result follows from Lemma 3.2.

(iii) The argument we use is similar. This time we have to control

$$
\int_{\mathscr{T}_{N_{t}}}^{t} g^{2}\left(X_{s}\right) d s
$$

Since $\mathbb{E}\left(\int_{\mathscr{T}_{N_{t}}}^{t} g^{2}\left(X_{s}\right) d s\right) \leq \sigma^{2}$, we conclude that for any $\theta>0$

$$
\lim _{t \rightarrow \infty} \frac{\int_{\mathscr{T}_{N_{t}}}^{t} g^{2}\left(X_{s}\right) d s}{t^{\theta}} \stackrel{\mathbb{P}}{=} 0
$$

Lemma 2.1 and Lemma 3.3 allow us to conclude.

(iv) In order to study the asymptotic distribution of $\frac{\int_{0}^{t} g\left(X_{s}\right) d B_{s}}{\sqrt{\int_{0}^{t} g^{2}\left(X_{s}\right) d s}}$, it is enough to study

$$
\frac{\sum_{i=0}^{N_{t}} \psi_{i}}{\sqrt{M_{t}}}
$$

The same type of argument used in the proof of Lemma 3.2, shows that the asymptotic distribution for this ratio is $N\left(0, \sigma^{2}\right)$. Then part (iv) allows us to finish the proof of Theorem 2.4.

3.5. General initial distribution. We shall show how to prove Theorem 2.4 under a general initial condition $X_{0}$, which we assume has a distribution independent of $B$. It is clear that it is enough to show the result for a non random initial condition $X_{0}=x$.

We shall give a sketch of the proof in case $x= \pm 1$. The other cases are similar. Let us prove $(i i)$, that is under both $\mathbb{P}_{1}, \mathbb{P}_{-1}$ we have

$$
\lim _{t \rightarrow \infty} \frac{\int_{0}^{t} g\left(X_{s}\right) d B_{s}}{t^{1 / 4}} \stackrel{\mathrm{d}}{=} \sigma \sqrt{\Gamma} Y
$$

We denote by $F$ the distribution of $\sigma \sqrt{\Gamma} Y$. The first thing to show is that

$$
\left(\frac{\int_{0}^{t_{k}} g\left(X_{s}\right) d B_{s}}{t_{k}^{1 / 4}}\right)_{k}
$$


is tight, for any sequence $t_{k} \uparrow \infty$, under both $\mathbb{P}_{1}, \mathbb{P}_{-1}$. This is a consequence of the fact

$$
\mathbb{P}_{1}\left(t^{-1 / 4}\left|\int_{0}^{t} g\left(X_{s}\right) d B_{s}\right|>z\right) \leq \frac{1}{p} \mathbb{P}\left(t^{-1 / 4}\left|\int_{0}^{t} g\left(X_{s}\right) d B_{s}\right|>z\right),
$$

where in the right hand side the initial distribution is the stationary distribution $(p, q)$. Then, tightness under $\mathbb{P}_{1}$ follows from tightness under $\mathbb{P}$. So we can assume that $t_{k}^{-1 / 4}\left|\int_{0}^{t_{k}} g\left(X_{s}\right) d B_{s}\right|$ is convergent (in distribution) to $F_{1}, F_{-1}$ under $\mathbb{P}_{1}, \mathbb{P}_{-1}$ respectively.

Consider that $X_{0}=1$ and define $T=\inf \left\{t>0: X_{t}=-1\right\}$. This is a finite stopping time and then

$$
\left|\mathbb{P}_{1}\left(t_{k}^{-1 / 4} \int_{0}^{t_{k}} g\left(X_{s}\right) d B_{s} \leq y\right)-\mathbb{P}_{1}\left(t_{k}^{-1 / 4} \int_{0}^{t_{k}} g\left(X_{s}\right) d B_{s} \leq y, T<t_{k}\right)\right| \leq \mathbb{P}_{1}\left(T>t_{k}\right) \rightarrow 0 .
$$

On the other hand, since $\int_{0}^{T} g\left(X_{s}\right) d B_{s}$ is a finite random variable, then

$$
t_{k}^{-1 / 4} \int_{0}^{T} g\left(X_{s}\right) d B_{s} \rightarrow 0 \text { a.s. }
$$

This observation shows that, if $y$ is a point of continuity for $F_{1}$ then

$$
F_{1}(y)=\lim _{k \rightarrow \infty} \mathbb{P}_{1}\left(t_{k}^{-1 / 4} \int_{T}^{t_{k}} g\left(X_{s}\right) d B_{s} \leq y, T<t_{k}\right)
$$

Given that $X_{T}$ is constant and $B_{T+\bullet}-B_{T}$ is independent of $T$ we get $T, X_{T+\bullet}$ are independent and

$$
\begin{aligned}
& \mathbb{P}_{1}\left(t_{k}^{-1 / 4} \int_{T}^{t_{k}} g\left(X_{s}\right) d B_{s} \leq y, T<t_{k}\right) \\
= & \int_{0}^{t_{k}} \mathbb{P}_{-1}\left(t_{k}^{-1 / 4} \int_{u}^{t_{k}} g\left(X_{s}\right) d B_{s} \leq y\right) \mathbb{P}_{1}(T \in d u) .
\end{aligned}
$$

For every fixed $u \in(0, \infty)$, the random variable $\int_{0}^{u} g\left(X_{s}\right) d B_{s}$ is finite a.s. and therefore $t_{k}^{-1 / 4} \int_{0}^{u} g\left(X_{s}\right) d B_{s}$ converges to 0 a.s.. Under $\mathbb{P}_{-1}$, the sequence $t_{k}^{-1 / 4} \int_{0}^{t_{k}} g\left(X_{s}\right) d B_{s}$ converges in distribution to $F_{-1}$. We conclude that for all $y$, continuity point of $F_{-1}$, it holds

$$
\mathbb{P}_{-1}\left(t_{k}^{-1 / 4} \int_{u}^{t_{k}} g\left(X_{s}\right) d B_{s} \leq y\right) \rightarrow F_{-1}(y)
$$

Thus, the Dominated Convergence Theorem implies that for all $y$, point of continuity of $F_{1}$ and $F_{-1}$ (in particular for a dense set of values), we have

$$
F_{1}(y)=\int_{0}^{\infty} F_{-1}(y) \mathbb{P}(T \in d u)=F_{-1}(y) .
$$

The conclusion is that $F_{1}=F_{-1}$. But, using what we have already proved in the case where the initial distribution of $X_{0}$ is the stationary distribution $(p, q)$, we get

$$
p F_{1}(y)+q F_{-1}(y)=F(y) .
$$

The conclusion is that $F_{1}=F_{-1}=F$. The limit point for $\left(t_{k}^{-1 / 4} \int_{0}^{t_{k}} g\left(X_{s}\right) d B_{s}\right)_{k}$, under $\mathbb{P}_{1}$ or $\mathbb{P}_{2}$, is independent of the subsequence, which implies that

$$
\left(t^{-1 / 4} \int_{0}^{t} g\left(X_{s}\right) d B_{s}\right)_{t} \rightarrow F
$$

under both $\mathbb{P}_{1}$ and $\mathbb{P}_{-1}$. 


\section{Applications}

This section is devoted to some applications of Theorem (2.4), first to estimate the parameter of the Skew Brownian motion with one or two barriers, and second to the nonparametric estimation of the drift coefficient in a stochastic differential equation.

We would like to highlight that based on the elements in the proofs given before, we can establish a simple way to simulate some functionals of the unique solution of (1.1). The simulation consists of two independent blocks: first generate independents paths of $X$ until the first time it leaves \pm 2 (for example, by Euler method, see Gobet, 2000 and references therein). Then, simulate an independent Markov chain $Z$ with states $\{1,-1\}$ and transition probabilities given by (2.2).

Remark 4.1. The length, $T_{i}$, of each renovation interval $\left(\mathscr{R}_{i}, \mathscr{T}_{i}\right)$ is simple to simulate. The density of this length is given by

$$
f(t)=\frac{1}{\sqrt{2 \pi t^{3}}} e^{-\frac{1}{2 t}}, \quad t \geq 0
$$

A possible scheme for simulation is found in Feller (1971), page 51.

4.1. Parameter Estimation in the Skew Brownian motion model. A skew Brownian motion (sBm) of parameter $\alpha$ is defined by the strong solution of the stochastic differential equations with local time given by

$$
X_{t}=x+B_{t}+(2 \alpha-1) L_{t}^{0}(X) .
$$

where $B_{t}$ is a Brownian motion, $\alpha \in[0,1]$ is constant and $L_{t}^{0}(X)$ is the symmetric local time at 0 of $X$. SBm is obtained from a Brownian motion by independently modifying the signs of the excursions away from zero, $\alpha$ is the probability that the excursion is positive. Notice that for $\alpha=0$ or $\alpha=1$ we obtain a Reflected Brownian motion, while for $\alpha=0.5$ we get a Bm. A survery of sBm can be found in Lejay (2006). The main purpose of this application is to obtain an estimation of the parameter $\theta=2 \alpha-1$.

Le Gall (1984) considers the problem of approximating the unique solution of a Stochastic Differential Equation (SDE in short) of the type

$$
X_{t}=X_{0}+\int_{0}^{t} f\left(X_{s}\right) d B_{s}+\int_{\mathbb{R}} \nu(d a) L_{t}^{a}(X)
$$

where $\nu$ is a bounded measure on $\mathbb{R}$. The sBm corresponds to the case $\nu=(2 \alpha-1) \delta_{0}\left(\delta_{0}\right.$ denotes the Dirac measure at 0$)$. On the other hand, if $\nu$ is absolutely continuous with respect to the Lebesgue measure $\nu(d a)=h(a) d a$, then $X$ satisfies

$$
X_{t}=X_{0}+\int_{0}^{t} f\left(X_{s}\right) d B_{s}+\int_{0}^{t}\left(h f^{2}\right)\left(X_{s}\right) d s .
$$

Under some regularity assumptions, Le Gall proved that any solution of (4.2) is the strong limit of a sequence of solutions of (4.3). Conversely, any limit of a sequence of solutions of (4.3) is a solution of (4.2).

In sBm case, we take $f=1$ and $h=\theta \ell$, where $\theta=2 \alpha-1$ and $\ell d x$ is an approximation of $\delta_{0}$. We are interested to the estimation of $\theta$, in this approximated model, when Least Square estimation procedure is used. In this case the error $\hat{\theta}_{L S}^{t}-\theta$ is given by

$$
\hat{\theta}_{L S}^{t}-\theta=\frac{\int_{0}^{t} h\left(X_{s}\right) d B_{s}}{\int_{0}^{t} h^{2}\left(X_{s}\right) d s} .
$$

Our results give the asymptotic distribution for this error, which is of the order $t^{-1 / 4}$. 
Considering the general framework in Le Gall (1984) and equations (4.2) and (4.3), we can approximate the Skew Brownian motion by means of the equation

$$
X_{t}=X_{0}+B_{t}+\theta \int_{0}^{t} \frac{n}{\sqrt{2 \pi}}\left(e^{-\frac{n^{2} X_{s}^{2}}{2}}-e^{-n^{2} / 2}\right) 1_{\left|X_{s}\right| \leq 1} d s .
$$

In the direction of to estimate the parameter $\theta$, we consider the following functional of $X$

$$
F_{t}=\int_{0}^{t} g\left(X_{s}\right) d B_{s}=\int_{0}^{t} \frac{n}{\sqrt{2 \pi}}\left(e^{-\frac{n^{2} X_{s}^{2}}{2}}-e^{-n^{2} / 2}\right) 1_{\left|X_{s}\right| \leq 1} d B_{s},
$$

where $t \in[0, T]$, and we assume $T=10000$. We approximate the solution of equation (4.5) by means of an Euler scheme, with step size discretization $T / M, M=100000, X_{0}=0$ and $\mathrm{n}=1000$. All simulations were carried out in Matlab software. These simulations should be taken with caution, because to approximate $\delta_{0}$ a finer should be carried out. Our simulations are intended to give only a rough overview of what is happening.

The Figure 4.2 presents a path of the solution of equation (4.5) for different values of $\theta$, recall that $\theta=0$ corresponds to standard Bm.

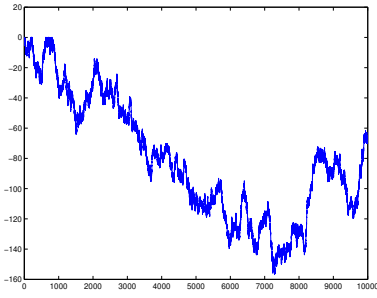

$\theta=-1$

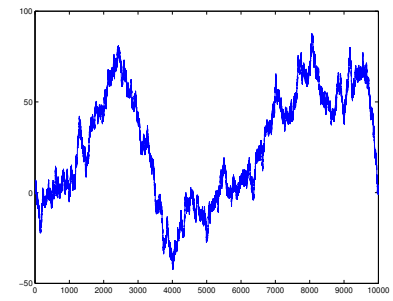

$\theta=0$

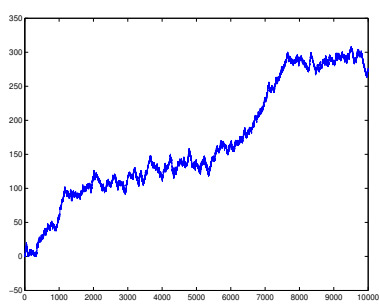

$$
\theta=1
$$

Figure 4.2. Path (4.5)

The Figure 4.3 presents the comparison between $\int_{0}^{T} g^{2}\left(X_{s}\right) d s$ (black line) and $c \sqrt{T}$ (light blue line) for equations (4.5) for different values of $\theta$.

To estimate the parameter $\theta=2 \alpha-1$, we will make use of the Least Square procedure. In this case the estimator $\hat{\theta}_{L S}^{T}(n)$ is given by:

$$
\hat{\theta}_{L S}^{T}(n)=\frac{\int_{0}^{T} g\left(X_{s}\right) d X_{s}}{\int_{0}^{T} g^{2}\left(X_{s}\right) d s}=\frac{\int_{0}^{T} \frac{n}{\sqrt{2 \pi}}\left(e^{-\frac{n^{2} X_{s}^{2}}{2}}-e^{-\frac{n^{2}}{2}}\right) 1_{\left|X_{s}\right| \leq 1} d X_{s}}{\int_{0}^{T}\left(\frac{n}{\sqrt{2 \pi}}\left(e^{-\frac{n^{2} X_{s}^{2}}{2}}-e^{-\frac{n^{2}}{2}}\right) 1_{\left|X_{s}\right| \leq 1}\right)^{2} d s},
$$




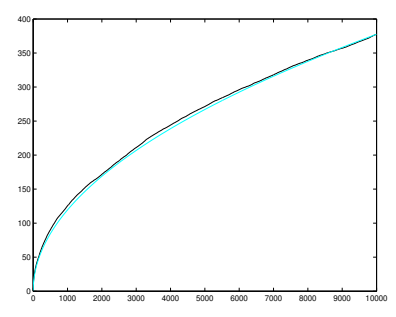

$\theta=-1$

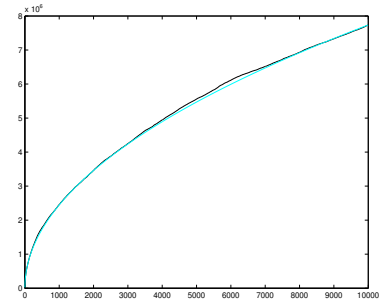

$\theta=0$

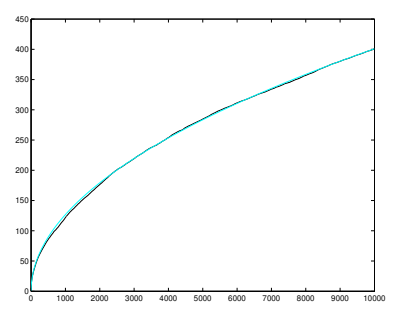

$\theta=1$

Figure 4.3. Comparison $\int_{0}^{T} g^{2}\left(X_{s}\right) d s$ vs $c \sqrt{T}$

and the error $\epsilon_{T}=\hat{\theta}_{L S}^{T}(n)-\theta$ is given by:

$$
\epsilon_{T}=\frac{\int_{0}^{T} g\left(X_{s}\right) d B_{s}}{\int_{0}^{T} g^{2}\left(X_{s}\right) d s}=\frac{\int_{0}^{T} \frac{n}{\sqrt{2 \pi}}\left(e^{-\frac{n^{2} X_{s}^{2}}{2}}-e^{-n^{2} / 2}\right) 1_{\left|X_{s}\right| \leq 1} d B_{s}}{\int_{0}^{T}\left(\frac{n}{\sqrt{2 \pi}}\left(e^{-\frac{n^{2} X_{s}^{2}}{2}}-e^{-\frac{n^{2}}{2}}\right) 1_{\left|X_{s}\right| \leq 1}\right)^{2} d s} .
$$

To the best of our knowledge, the only works on the estimation for the skew parameter are given in Lejay et al. (2014, 2019), these are based on the Maximum Likelihood estimation (ML), and after that there is no similar results in this direction.

By Theorem 2.4 the error $\sigma T^{1 / 4} \epsilon_{T}$ converges to the ratio of a standard Normal distribution and an independent $\sqrt{\Gamma}$.

The next table, shows the estimation of different values of $\theta$. We use $N=1000$ samples, with $n=1000$.

\begin{tabular}{|c|c|c|}
\hline$\theta_{L S}^{n}$ & Mean & Standard Deviation \\
\hline \hline 1 & 1.0062 & 0.0821 \\
\hline 0.5 & 0.5043 & 0.0844 \\
\hline 0 & $-1.3339 e^{-05}$ & $7.8772 e^{-04}$ \\
\hline-0.5 & -0.5088 & 0.0874 \\
\hline-1 & -1.0059 & 0.1008 \\
\hline
\end{tabular}

TABLE 4.1. Estimation of different values of $\theta$.

The Figure 4.4 shows the histograms of the error $\epsilon_{T}$, for $N=1000$ samples. We see that the standard deviation is of order $\frac{1}{T^{1 / 4}}$, except in the case $\theta=0$ that corresponds to the standard Brownian motion. All simulations were carried out in Matlab software. 


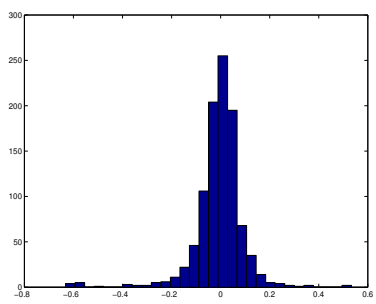

$\theta=-1$

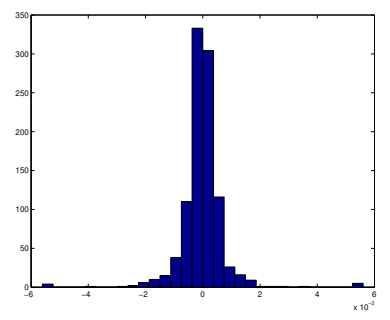

$\theta=0$

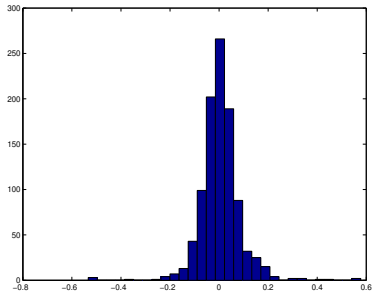

$\theta=1$

Figure 4.4. Histograms for different values of $\theta$.

\section{Simple versus simple hypothesis testing}

We assume the null hypothesis $H_{0}$ and the alternative hypothesis $H_{1}$ given by

$$
H_{0}: \theta=\theta_{0} \text { versus } H_{1}: \theta=\theta_{1}
$$

for some $\theta_{0}, \theta_{1} \in[-1,1]$, with $\theta_{1}>\theta_{0}$.

Test $\varphi: T^{\frac{1}{4}} \sigma(\hat{\theta}-\theta)$. By Theorem 2.4, $\lim _{T \rightarrow \infty} \varphi \stackrel{\mathrm{d}}{=} \Gamma^{-\frac{1}{2}} Y$, where $\Gamma$ has the distribution of the absolute value of a $N(0,1), Y$ is a $N(0,1)$, both independent; and $\sigma$ is given by (2.8).

By a direct calculus, we have that the density function of the distribution $\Gamma^{-\frac{1}{2}} Y$ is given by

$$
f(t)=\frac{1}{\sqrt{\pi} 2^{7 / 4}} e^{t^{2} / 8} D_{-\frac{3}{2}}\left(\frac{t^{2}}{\sqrt{2}}\right),
$$

where $D$ is the parabolic cylinder function given by

$$
D_{p}(z)=\frac{e^{-z^{2} / 4}}{\Gamma(-p)} \int_{0}^{\infty} e^{-x z-x^{2} / 2} x^{-p-1} d x
$$

for $p<0$.

Let $\gamma=\mathbb{P}\left\{\right.$ reject $\left.H_{0} \mid \theta_{0}\right\}$, and $\beta=\mathbb{P}\left\{\right.$ fail to reject $\left.H_{0} \mid \theta_{1}\right\}$.

For example, since the density function of $\Gamma^{-\frac{1}{2}} Y$ is symmetric, choosing a small $\gamma$, the critical region is as shown in the following figure 4.5 then we will reject $H_{0}$ for any value of $\theta>x$. The critical value $x$ is calculated such that

$$
\gamma=\mathbb{P}\left\{\hat{\theta}>x \mid \theta_{0}\right\}=\mathbb{P}\left\{T^{\frac{1}{4}} \sigma\left(\hat{\theta}-\theta_{0}\right)>T^{\frac{1}{4}} \sigma\left(x-\theta_{0}\right)\right\}=\mathbb{P}\left\{\Gamma^{-\frac{1}{2}} Y>T^{\frac{1}{4}} \sigma\left(x-\theta_{0}\right)\right\},
$$

where $\sigma$ given by (2.8) is obtained first taking $N$ simple paths $X\left(\omega_{1}\right), \cdots, X\left(\omega_{N}\right)$ of process $X$, calculate the times $R_{1}\left(\omega_{i}\right)(i=1, \cdots N)$ and by Monte Carlo method, approximate the integral $\int_{0}^{R_{1}\left(\omega_{i}\right)} g^{2}\left(X_{s}\left(\omega_{i}\right)\right) d s$ for the function $g$ given in (4.6). Finally,

$$
\sigma \approx \frac{1}{N} \sum_{i=1}^{N} \int_{0}^{R_{1}\left(\omega_{i}\right)} g^{2}\left(X_{s}\left(\omega_{i}\right)\right) d s .
$$




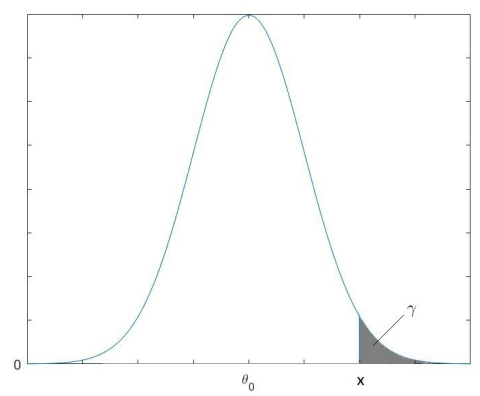

FiguRE 4.5. Critical region

Skew Brownian motion with two barriers Now we consider the Skew Brownian motion with two barriers (see Ouknine, 1990; Ramirez, 2011) given by

$$
X_{t}=x+B_{t}+\left(2 \alpha_{1}-1\right) L_{t}^{x_{1}}(X)+\left(2 \alpha_{2}-1\right) L_{t}^{x_{2}}(X),
$$

where $L_{t}^{x_{i}}(X)$ denotes the local time at $x_{i}$ for the time $t$ of the process $X, \alpha_{1}, \alpha_{2} \in[0,1]$ and barriers $x_{1}<x_{2}$. The term $\alpha_{i}$ is called the skewness at the barrier $x_{i}(i=1,2)$. In Ramirez (2011), the author proved that the paths of this process behave as those of Brownian motion when away from the barriers; and the process is skewed by $\alpha_{i}$ at barrier $x_{i}$, for $i=1,2$. The sBm with two barriers corresponds to the case $\nu=\left(2 \alpha_{1}-1\right) \delta_{x_{1}}+\left(2 \alpha_{1}-1\right) \delta_{x_{2}}$ in equation (4.2).

We are interested to estimate the parameters $\theta_{i}=2 \alpha_{i}-1(i=1,2)$ by Least Square method. Without loss of generality we shall assume that $x_{1}, x_{2} \in(0,1)$. Similarly as the skew Brownian motion, it is possible approximate the process $\left\{X_{t}\right\}$ by the solutions of equations

$$
X_{t}^{(n)}=x+B_{t}+\theta_{1} \int_{0}^{t} g_{1}^{(n)}\left(X_{s}\right) 1_{\left\{a_{1} \leq X_{s} \leq b_{1}\right\}} d s+\theta_{2} \int_{0}^{t} g_{2}^{(n)}\left(X_{s}\right) 1_{\left\{a_{2} \leq X_{s} \leq b_{2}\right\}} d s,
$$

where $g_{i}^{(n)}\left(X_{s}\right) 1_{\left\{a_{i} \leq X_{s} \leq b_{i}\right\}}$ is an approximation of $\delta_{x_{i}}$ for $i=1,2\left(\delta_{x}\right.$ denotes the Dirac measure at $\mathrm{x}), n \in \mathbb{N}$ and $-1<a_{1}<b_{1}<a_{2}<b_{2}<1$. Like in the case of the sBm, we can take

$$
g_{i}(x)=\left(e^{-\frac{n^{2}\left(X_{s}-x_{i}\right)^{2}}{2}}-e^{-\frac{n^{2}}{2}}\right), \quad i=1,2 .
$$

Note that since $1_{\left\{a_{1} \leq X_{s} \leq b_{1}\right\}} 1_{\left\{a_{2} \leq X_{s} \leq b_{2}\right\}}=0$, then the Least square estimator $\hat{\theta}_{i}^{T}(n)$ of $\theta_{i}$ and the error $\epsilon_{T}^{(i)}=\hat{\theta}_{i}^{T}(n)-\theta_{i}$, for $i=1,2$, are given by

$$
\hat{\theta}_{i}^{T}(n)=\frac{\int_{0}^{T} g_{i}^{(n)}\left(X_{s}\right) 1_{\left\{a_{i} \leq X_{s} \leq b_{i}\right\}} d X_{s}}{\int_{0}^{T}\left(g_{i}^{(n)}\left(X_{s}\right)\right)^{2} 1_{\left\{a_{i} \leq X_{s} \leq b_{i}\right\}} d s},
$$

and

$$
\epsilon_{T}^{(i)}=\frac{\int_{0}^{T} g_{i}^{(n)}\left(X_{s}\right) 1_{\left\{a_{i} \leq X_{s} \leq b_{i}\right\}} d B_{s}}{\int_{0}^{T}\left(g_{i}^{(n)}\left(X_{s}\right)\right)^{2} 1_{\left\{a_{i} \leq X_{s} \leq b_{i}\right\}} d s} .
$$

Hence, from Theorem 2.4

$$
\lim _{T \rightarrow \infty} T^{\frac{1}{4}} \epsilon_{T}^{(i)} \stackrel{\mathrm{d}}{=} \sigma_{i}^{-1} \Gamma^{-\frac{1}{2}} Y, \quad i=1,2
$$

where

$$
\sigma_{i}^{2}=\mathbb{E}_{X_{0}}\left(\int_{0}^{R_{1}}\left(g_{i}^{(n)}\left(X_{s}\right)\right)^{2} 1_{\left\{a_{i} \leq X_{s} \leq b_{i}\right\}} d s\right)
$$


Remark 4.2. (1) If one or both barriers do not belong to $[-1,1]$, we consider a bigger interval with the both barriers and take appropriate functions $g_{1}, g_{2}$ with a support contained in the new interval.

(2) The same technique above could be apply for sBm with more than two barriers.

\subsection{Nonparametric estimation of the drift coefficient in SDE.}

We are interested in the non-parametric estimation of $h(x)$ for a fixed point $x$ in (1.1) based on the observation $X_{t}, t \in[0, T]$. In Tuan (1981) the estimators for $h(x)$ are given by

$$
\hat{h}_{T}(x)=\frac{\int_{0}^{T} K\left(\frac{X_{t}-x}{b_{T}}\right) d X_{t}}{\int_{0}^{T} K\left(\frac{X_{t}-x}{b_{T}}\right) d t},
$$

where $K$ is a non-negative real kernel function such that $\int_{\mathbb{R}} K(z) d z=1$. In Löcherbach and Loukianova (2008) the authors consider the asymptotic of these estimators in the context of drift estimation for 1-D diffusions. In the same vain, we assume the Kernel $K$ has compact support contained in $[-1,1]$ and the bandwidth parameter $b_{T}$ is constant. Some examples of Kernels are

- The triangle kernel

- The parabolic kernel

$$
K(x)=(1-|x|) 1_{[-1,1]}(x) .
$$

- The quartic kernel

$$
K(x)=\frac{3}{4}\left(1-x^{2}\right) 1_{[-1,1]}(x)
$$

$$
K(x)=\frac{15}{16}\left(1-x^{2}\right) 1_{[-1,1]}(x) .
$$

The error $\epsilon_{T}=\hat{h}_{T}(x)-h(x)$ is given by:

$$
\epsilon_{T}=\frac{\int_{0}^{T} K\left(\frac{X_{t}-x}{b_{T}}\right) d B_{t}}{\int_{0}^{T} K\left(\frac{X_{t}-x}{b_{T}}\right) d t}
$$

From Theorem 2.4, first replacing the function $g$ by the Kernel $K$,

$$
\lim _{T \rightarrow \infty} \frac{\int_{0}^{T} K\left(\frac{X_{s}-x}{b_{T}}\right) d B_{s}}{T^{1 / 4}} \stackrel{\mathrm{d}}{=} \sigma_{1} \sqrt{\Gamma} Y,
$$

where $\Gamma$ has the distribution of the absolute value of a $N(0,1), Y$ is a $N(0,1)$ and

$$
\sigma_{1}^{2}=\mathbb{E}_{X_{0}}\left(\int_{0}^{R_{1}} K^{2}\left(\frac{X_{s}-x}{b_{T}}\right) d s\right)
$$

with $R_{1}$ is the first hitting time of $\left\{x-b_{T}-1, x+b_{T}+1\right\}$ from the previous visit to $\left\{x-b_{T}, x+b_{T}\right\}$ (see 2.1).

Now, taking the function $g=K^{\frac{1}{2}}$, we obtain

$$
\lim _{T \rightarrow \infty} \frac{\int_{0}^{T} K\left(\frac{X_{s}-x}{b_{T}}\right) d s}{M_{T}} \stackrel{\mathbb{P}}{=} \sigma_{2}^{2},
$$

where

$$
\sigma_{2}^{2}=\mathbb{E}_{X_{0}}\left(\int_{0}^{R_{1}} K\left(\frac{X_{s}-x}{b_{T}}\right) d s\right)
$$

From (4.13), (4.14) and Theorem 2.4 (i), we have that

$$
\lim _{T \rightarrow \infty} T^{\frac{1}{4}} \epsilon_{T} \stackrel{\mathrm{d}}{=} \frac{\sigma_{1}}{\sigma_{2}} \Gamma^{-\frac{1}{2}} Y .
$$




\section{Notations}

We include a list of notations to facilitate the reading of this article.

- $\left(T_{i}\right)_{i}$ hitting times of $\{-1,1\}$ from the previous visit to $\{-2,2\}$ (see 2.1), with associated partial sums $\left(\mathscr{S}_{n}\right)$ and renewal process $\left(M_{t}\right)_{t}$ (see (2.6)).

- $\left(R_{i}\right)_{i}$ hitting times of $\{-2,2\}$ from the previous visit to $\{-1,1\}$ (see 2.1).

- $\left(\mathscr{T}_{i}\right)$ accumulated sums of $\left(R_{k}+T_{k}\right)$ until $i$, which correspond to the times of successive visits to $\{-1,1\}$ (see 2.1) and renewal process $\left(N_{t}\right)_{t}$ (see 2.5).

- $\left(\mathscr{R}_{i}\right)$ correspond to the times of successive visits to $\{-2,2\}$ (see 2.1).

- $\left(Z_{n}\right)_{n}$ is the Markov chain associated to the visits at $\{-1,1\}$, that is, $Z_{n}=X_{\mathscr{T}_{n}}$.

- $\mathscr{M}_{t}=\max _{0 \leq s \leq t} W_{s}$, the running maximum of the $\operatorname{Bm}\left(W_{t}\right)_{t}$.

\section{Acknowledgements}

The authors thank an anonymous referee for his/her helpful comments and references that have improved the presentation of this article.

\section{References}

Basawa, I. V. and Prakasa Rao, B. L. S. Statistical inference for stochastic processes. Academic Press, Inc. [Harcourt Brace Jovanovich, Publishers], London-New York (1980). ISBN 0-12-0802503. MR586053.

Delattre, S. and Hoffmann, M. Asymptotic equivalence for a null recurrent diffusion. Bernoulli, 8 (2), 139-174 (2002). MR1895888.

Delattre, S. and Jacod, J. A central limit theorem for normalized functions of the increments of a diffusion process, in the presence of round-off errors. Bernoulli, 3 (1), 1-28 (1997). MR1466543.

Feller, W. An introduction to probability theory and its applications. Vol. II. John Wiley \& Sons, Inc., New York-London-Sydney, second edition (1971). MR0270403.

Gobet, E. Weak approximation of killed diffusion using Euler schemes. Stochastic Process. Appl., 87 (2), 167-197 (2000). MR1757112.

Höpfner, R. and Löcherbach, E. Limit theorems for null recurrent Markov processes. Mem. Amer. Math. Soc., 161 (768), vi+92 (2003). MR1949295.

Jacod, J. and Shiryaev, A. N. Limit theorems for stochastic processes, volume 288 of Grundlehren der Mathematischen Wissenschaften [Fundamental Principles of Mathematical Sciences]. SpringerVerlag, Berlin, second edition (2003). ISBN 3-540-43932-3. MR1943877.

Khasminskii, R. Limit distributions of some integral functionals for null-recurrent diffusions. Stochastic Process. Appl., 92 (1), 1-9 (2001). MR1815176.

Khasminskii, R. Stochastic stability of differential equations, volume 66 of Stochastic Modelling and Applied Probability. Springer, Heidelberg, second edition (2012). ISBN 978-3-642-23279-4. MR2894052.

Kurtz, T. G. and Protter, P. Weak limit theorems for stochastic integrals and stochastic differential equations. Ann. Probab., 19 (3), 1035-1070 (1991). MR1112406.

Kutoyants, Y. A. Statistical inference for ergodic diffusion processes. Springer Series in Statistics. Springer-Verlag London, Ltd., London (2004). ISBN 1-85233-759-1. MR2144185.

Le Gall, J.-F. One-dimensional stochastic differential equations involving the local times of the unknown process. In Stochastic analysis and applications (Swansea, 1983), volume 1095 of Lecture Notes in Math., pp. 51-82. Springer, Berlin (1984). MR777514. 
Lejay, A. On the constructions of the skew Brownian motion. Probab. Surv., 3, 413-466 (2006). MR2280299.

Lejay, A., Mordecki, E., and Torres, S. Is a Brownian motion skew? Scand. J. Stat., 41 (2), 346-364 (2014). MR3207175.

Lejay, A., Mordecki, E., and Torres, S. Two consistent estimators for the skew Brownian motion. ESAIM Probab. Stat., 23, 567-583 (2019). MR3991913.

Löcherbach, E. and Loukianova, D. On Nummelin splitting for continuous time Harris recurrent Markov processes and application to kernel estimation for multi-dimensional diffusions. Stochastic Process. Appl., 118 (8), 1301-1321 (2008). MR2427041.

Ouknine, Y. Le "Skew-Brownian motion" et les processus qui en dérivent. Teor. Veroyatnost. $i$ Primenen., 35 (1), 173-179 (1990). MR1050069.

Prakasa Rao, B. L. S. Statistical inference for diffusion type processes, volume 8 of Kendall's Library of Statistics. Edward Arnold, London; Oxford University Press, New York (1999). ISBN 0-34074149-X. MR1717690.

Prakasa Rao, B. L. S. and Rubin, H. Asymptotic theory for process least squares estimators for diffusion processes. Technical Report, Purdue University (1979).

Ramirez, J. M. Multi-skewed Brownian motion and diffusion in layered media. Proc. Amer. Math. Soc., 139 (10), 3739-3752 (2011). MR2813404.

Revuz, D. and Yor, M. Continuous martingales and Brownian motion, volume 293 of Grundlehren der Mathematischen Wissenschaften [Fundamental Principles of Mathematical Sciences]. Springer-Verlag, Berlin (1991). ISBN 3-540-52167-4. MR1083357.

Takanobu, S. On the uniqueness of solutions of stochastic differential equations with singular drifts. Publ. Res. Inst. Math. Sci., 22 (5), 813-848 (1986). MR866659.

Tuan, P. D. Nonparametric estimation of the drift coefficient in the diffusion equation. Math. Operationsforsch. Statist. Ser. Statist., 12 (1), 61-73 (1981). MR607955. 